\title{
Chemical Polymerization of Aniline on a Polystyrene Sulfonate Membrane: Controlling the Polymerization Site Using Different Oxidants
}

\author{
Sophie Tan, Jacqueline H. Tieu and Daniel Bélanger ${ }^{*}$ \\ Département de Chimie, Université du Québec à Montréal, Case Postale 8888, succursale \\ Centre-Ville, Montréal (Québec) Canada H3C 3P8
}

\section{Supporting Information}

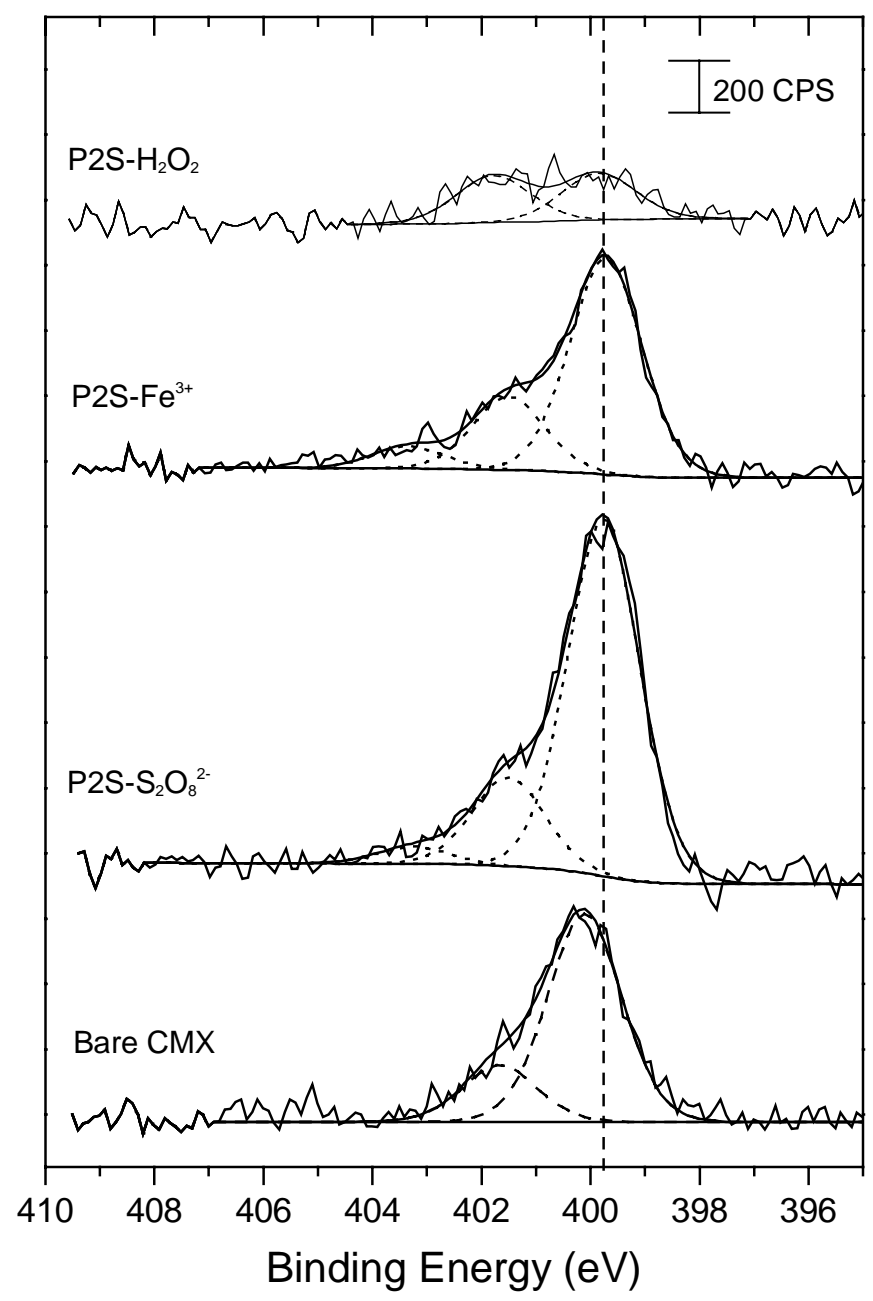

Figure S1. N1s core level XPS spectra of the bare and modified CMX membranes using the two-step method. The surface shown is the side that was exposed to the reagents. The deconvolution of $\mathrm{P} 2 \mathrm{~S}-\mathrm{S}_{2} \mathrm{O}_{8}{ }^{2-}$ and $\mathrm{P} 2 \mathrm{~S}-\mathrm{Fe}^{3+}$ do not include the CMX nitrogen contribution. 


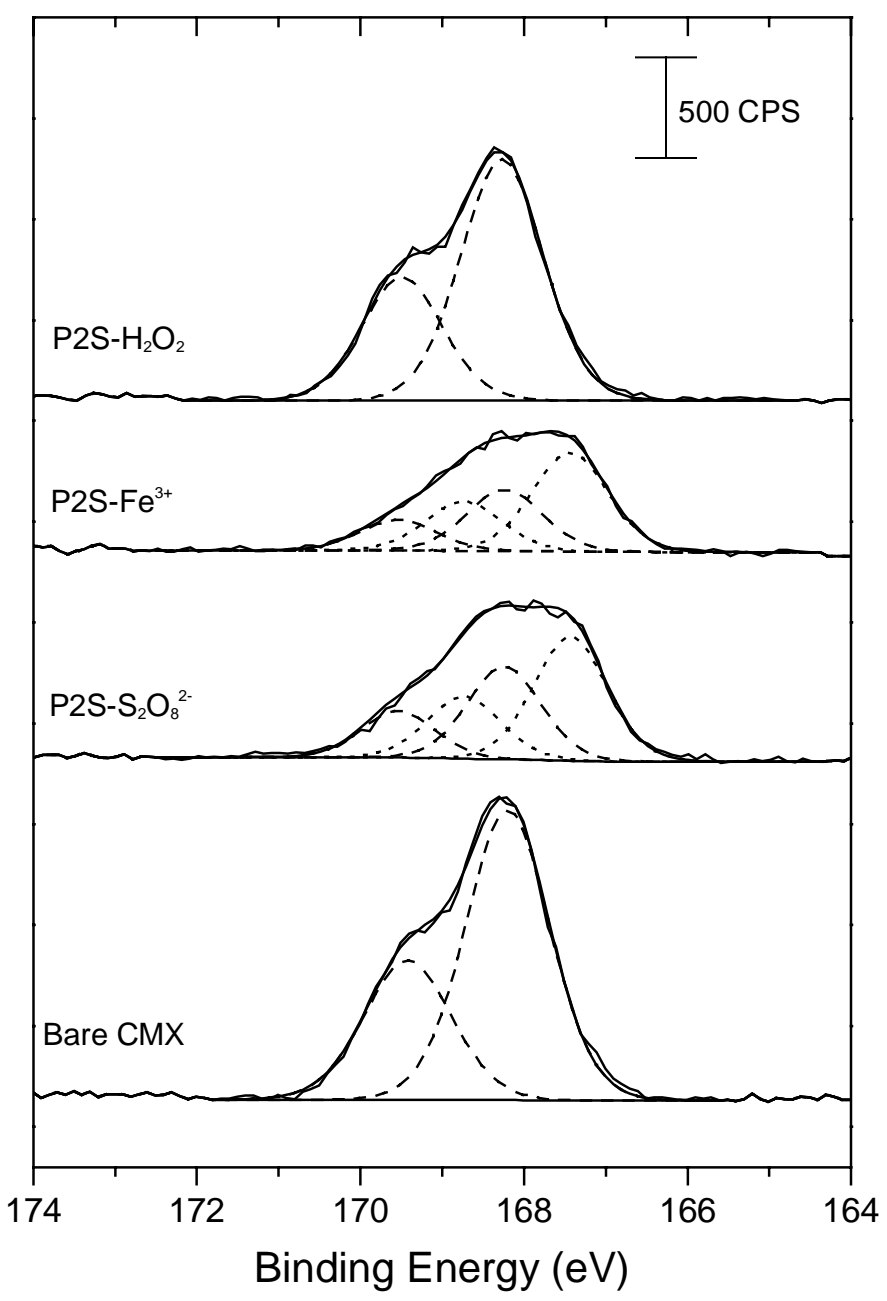

Figure S2. Deconvoluted S2p core level spectra of the bare and modified CMX membranes using the two-step method where (...) represents the $\mathrm{SO}_{3}{ }^{-}$groups interacting with $\mathrm{PANI}^{+}$and (---), the $\mathrm{SO}_{3}^{-}$with $\mathrm{H}^{+}$counterion. The surface shown is the side that was exposed to the oxidant during the modification procedures. 
Table S1. XPS and elemental analysis results of membranes treated solely with the oxidant using the one-step method (1 hour reaction).

\begin{tabular}{lcccc}
\hline & XPS & & \multicolumn{2}{c}{ Elemental Analysis } \\
& \% at. $\mathbf{~}$ & \% at. $\mathbf{S}$ & \% wt. $\mathbf{~}$ & \% wt. S \\
\hline Bare $\mathrm{CMX}^{2-}$ & 3.4 & 5.0 & $0.61 \pm 0.07$ & $5.1 \pm 0.6$ \\
$\mathrm{CMX} \mathrm{S}_{2} \mathrm{O}_{8}{ }^{2-}$ & 2.0 & 5.0 & 0.60 & 4.9 \\
$\mathrm{CMX} \mathrm{Fe}^{3+}$ & 2.4 & 5.4 & 0.57 & 5.0 \\
$\mathrm{CMX} \mathrm{H}_{2} \mathrm{O}_{2}$ & 2.0 & 4.8 & 0.54 & 5.3 \\
\hline
\end{tabular}

